\title{
FT-IR Microspectroscopic Imaging of Cross-Sectioned Human Hair during a Bleaching Process
}

\author{
Soo Ryeon Ryu1, Wonhyeong Jang1, Si-In Yu², Byeong-Ha Lee², Oh-Sun Kwon', Kwanwoo Shin ${ }^{1 *}$ \\ ${ }^{1}$ Department of Chemistry, Institute of Biological Interfaces, Sogang University, Seoul, South Korea \\ ${ }^{2}$ Department of Life Science, Sogang University, Seoul, South Korea \\ Email: *kwshin@sogang.ac.kr
}

How to cite this paper: Ryu, S.R., Jang, W., Yu, S.-I., Lee, B.-H., Kwon, O.-S. and Shin, K. (2016) FT-IR Microspectroscopic Imaging of Cross-Sectioned Human Hair during a Bleaching Process. Journal of Cosmetics, Dermatological Sciences and Applications, 6, 181-190.

http://dx.doi.org/10.4236/jcdsa.2016.65023

Received: August 22, 2016

Accepted: October 14, 2016

Published: October 17, 2016

Copyright $\odot 2016$ by authors and Scientific Research Publishing Inc. This work is licensed under the Creative Commons Attribution International License (CC BY 4.0).

http://creativecommons.org/licenses/by/4.0/

\begin{abstract}
We investigated bleached human hair by FT-IR microspectroscopy and chemical imaging. The cross sectioned hair is approximately $90 \mu \mathrm{m}$ in diameter, showed cuticle and cortex in chemical imaging. Differential amide I/II absorbance ratio and broadening amide I band between in the cortex and cuticle were confirmed in FT-IR microspectroscopy and chemical imaging. The cystine monoxide band from the products of disulfide oxidation of the amino acid cystine is associated with hair damaging during bleaching process. With increase bleaching time, the band for cystine monoxide shows more intense and larger area in chemical image. The spatially chemical change was investigated in detail by FT-IR microspectroscopy and chemical imaging during the bleached process.
\end{abstract}

\section{Keywords}

FT-IR Imaging, Human Hair, Bleaching

\section{Introduction}

A human hair is approximately $50-100 \mu \mathrm{m}$ in diameter and consists of a barrier cuticle layer (outer $5 \mu \mathrm{m}$ ), a middle cortex layer $(40-95 \mu \mathrm{m}$ ), and, when present, an inner medulla $(5-10 \mu \mathrm{m})$. A tightly packed hair fiber is composed of crystalline keratin, mostly in an $\alpha$-helical arrangement [1] [2]. The cuticle protects the cortex from the attacks suffered by hair, and the hair's cosmetic properties, similar to the "hand" of a finished textile (i.e., stiffness, smoothness, fullness and softness, anti-drape stiffness, crispness, flexibility etc.), depend strongly on the condition of the cuticle. Therefore, most studies in cosmetics have focused on the physical and the visual properties of the 
cuticle's surface. However, the cortex represents $90 \%$ of the hair fiber's total weight and gives the hair fiber its remarkable properties. The cortex is richer in leucine, glutamic acid and the amino acids that are generally found in an $\alpha$-helical protein. Moreover, the cortex is richer in diacidic amino acids, lysine, and histidine than the cuticle is [2]. Nevertheless, studies on the cortex are lacking. In particular, the color of human hair is due to the presence of melanin, and grains of melanin are mostly found in the cortex [2] [3].

Hair analysis has been successfully used in many areas such as forensic, cosmetic, and medical sciences because it often reflects the health condition of a human being. Typically in forensic science, hair analyses are performed by using microscopic examination and identification. A microscopic analysis provides information about the length, color, damage, and other characteristics of both human and animal hair [4]. Even though microscopic analyses are widely used in forensic science to determine the specific properties of hair to be used as evidence [5] [6], these techniques when limited to surface analyses often have inherent flaws. By using spectroscopy, one is able to obtain, based on the chemistry of hair, additional information reflecting the chemical and the biological characteristics. The analysis of evidence by using spectroscopy often applies not only to information about natural hair but also to manmade traces such as gunshot residue or dyes [4] [7]. In toxicology, hair analysis is further used to provide evidence of heavy-metal poisoning (lead, cadmium, arsenic, and mercury) and the presence of drugs (cocaine, heroin, and amphetamines) [8]. Recently, Kakoulli et al. reported the distribution of arsenic in ancient human hair by using synchrotron radiation [8]. Furthermore, in recent years, hair has been a widely accepted biomonitor in medical science; e.g., hair from cancer patients has been compared with hair from healthy individuals in attempts to identify possible applications in cancer research and diagnosis [9]-[11].

Hair is known not to be able to restore itself as other organs and cells can. Hence, many people want to maintain their hair in a natural and beautiful condition, so in cosmetic science, many products have been developed to prevent hair from being damaged or to repair it when it has been damaged [12]-[14]. The change in the conformation of hair during the damage process has been studied to provide information on the features of damaged hair [13]-[15]. Although the cuticle and the cortex in hair have different conformations, most studies have focused on the cuticle [13] [14]. In particular, because many people bleach their hair, many researchers have studied bleached hair to reduce hair damage cause by bleaching agents. Because grains of melanin are eliminated during the bleaching process, Wolfram and coworkers studied the mechanism of hair bleaching with a focus on both the melanin granules in hair and the changes in the chemical composition of the entire hair [3].

The majority of vibrational spectroscopic studies have been carried out to obtain averaged data from a sample by examining the vibrational modes of its components. Conventional FT-IR spectroscopy only provides information regarding the sample's chemical composition, but a FT-IR analysis can provide rapid and specific chemical in- 
formation at the molecular level [16]. Thus, the IR spectroscopic imaging technique has become a powerful tool in chemical analysis; IR microspectroscopic (IMS) imaging combines spectral and spatial information at the same time and may allow the distribution of vibrational modes on the surface to be visualized. IMS imaging is especially valuable when rough surfaces, such as febrile substrates (e.g., cellulose), are investigated because it also provides information about the surface's topography [17]. IMS imaging can also detect subtle chemical changes in tissues and cells without the use of a staining or a detecting agent [17]; it is a chemical imaging technique that can be applied to a broad range of samples and systems [17]-[19].

In 2000, Bantignes et al. first introduced IMS imaging with a synchrotron source. Chemical imaging showed a bonded water band within the cuticle as an effect of bleaching [12]. Since then, IMS imaging instrumentation has developed rapidly, and improved resolution and accessibility have been achieved even with a table-top source. For IMS imaging with very bright synchrotron radiation, apertures with sizes down to the diffraction limit of $\sim 3 \mu \mathrm{m}$ and with reasonable signal-to-noise ratios have been achieved. Recently, table top IR spectrometers equipped with an MCT focal plane array (FPA) detector or a linear MCT detector array with a moving sample stage have allowed the incoming beam through a large sample area to be spatially resolved with a high spatial resolution of $<1.56 \mu \mathrm{m} \times 1.56 \mu \mathrm{m}$. The development of the table top IMS system has provided easy access to this technique and has allowed changes to be observed as a function of time and under various conditions. Herein, spatial information obtained from the cortex and the cuticle during bleaching is presented. IMS imaging was used to provide information on how the oxidative agents in bleaches are diffused throughout the cortex and how the chemical reactions in the cortex, related to the bleaching, occur as a function of the bleaching time.

\section{Experimental}

Hair samples were collected from virgin, dark hair obtained from a South Korean male aged 20 years, which is presumed to be robust and healthy. The hair was cut into 10- to $15-\mathrm{cm}$ lengths starting approximately $5 \mathrm{~cm}$ from the root [20]. Each segment was then embedded in paraffin resin and sliced with a microtome to yield $10-\mu \mathrm{m}$-thick cross sections. The bleaching process was carried out at room temperature with $6 \%$ hydrogen peroxide $\left(6 \% \mathrm{w} / \mathrm{v} \mathrm{H}_{2} \mathrm{O}_{2}\right)$. The bleaching time was varied from 30 to $120 \mathrm{~min}$ in intervals of $30 \mathrm{~min}$. The interval of $30 \mathrm{~min}$ was chosen, because the bleaching process was confirmed clearly after first $30 \mathrm{~min}$ with naked eyes. The bleached samples were rinsed with deionized water and left to dry at ambient room temperature.

A FT-IR microspectrosocope (Agilent Cary 620 spectrometer) equipped with a mercury-cadmium-telluride (MCT) detector cooled with liquid nitrogen and a $16 \times 16$ focal plane array detector (FPA) was used to obtain all spectra with a spatial resolution of 5.5 $\mu \mathrm{m} \times 5.5 \mu \mathrm{m}$. All micro-spectra were collected in the transmission mode. The spectra were recorded with an $8-\mathrm{cm}^{-1}$ spectral resolution, and 128 interferograms were coadded for each measurement to ensure a high signal-to-noise ratio. For the analyses of 
the micro-spectra of the cross-sectioned hair, pre-treatments, such as a baseline correlation and smoothing, were carried out by using the Agilent resolution pro (Agilent, Santa Clara, CA) and the Origin8 (Originlab Corp., Northampton, MA) programs. Visual images were then analyzed by using the Image J program.

\section{Results and Discussion}

Human hair mostly consists of fibrous structural proteins, mainly keratin, so a strong amide I absorbance peak due to the $\mathrm{C}=\mathrm{O}$ vibration is expected at $\nu \sim 1658 \mathrm{~cm}^{-1}$, which can be assigned to the $\alpha$-helical structure [21] [22]. Figure 1 shows (A) an optical image (left) of a cross section of a human hair fixed in a paraffin block and a chemical image (right) of the amide I band for the cortex, cuticle, and paraffin block and (B) FTIR spectra (left) from the cortex, cuticle, and paraffin block obtained at $1658 \mathrm{~cm}^{-1}$ for the amide I band distribution of the cross-sectioned human hair and enlargements of the amide I and II regions (right) in the FT-IR spectra from the cortex and the cuticle. The color bar for the absorbance peak image from blue to red represents the intensity from
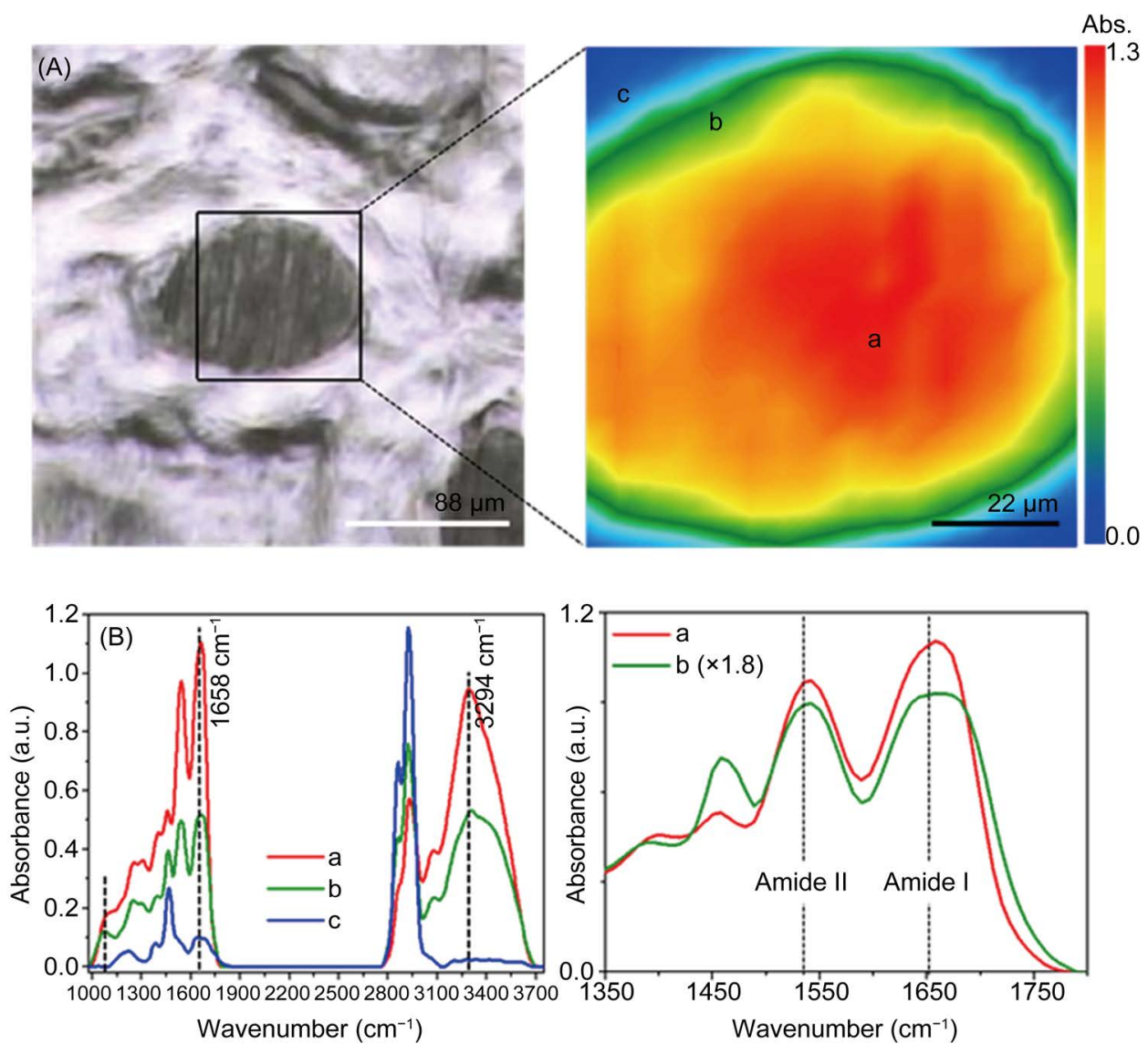

Figure 1. (A) (left) Optical image of a cross section of a human hair (dark area) fixed in a paraffin block (white area) and(right) chemical images of the amide I bands for the (a) cortex, the (b) cuticle, and the (c) paraffin block. (B) FT-IR spectra from the (a) cortex, the (b) cuticle, and the (c) paraffin block marked in the chemical image. Enlargements of the amide I and II regions in the spectra from the (a) cortex and the (b) cuticle are shown. 
background to the highest absorbance intensity at $1658 \mathrm{~cm}^{-1}$. Notably, the cuticle region surrounding the cortex can clearly be distinguished from the cortex by the green color in the chemical image, indicating a lack of the $\alpha$-helical form of proteins in that region. In addition, the cortex ( $\mathrm{a}$ in $\mathrm{A}$ ) with highest absorbance has a red color, the cuticle ( $b$ in $A$ ) with reduced absorbance has a green color, and the paraffin block ( $c$ in A) used to fix the hair for the cross-sectioning process has a blue color. The thickness of the cuticle band was ca. 3 - $5 \mathrm{~nm}$, which is in good agreement with data reported in the literature [2].

FT-IR spectra corresponding to the locations labeled a, b, and c in Figure 1(A) are shown on the left in Figure 1(B) and enlargements of the amide I and II regions in the spectra on the left are shown on the right. The spectra indicate that the pristine hair has an $\alpha$-helical protein conformation at the locations of both the cortex (a) and the cuticle (b), given that the amide I band, mainly associated with the $\mathrm{C}=\mathrm{O}$ stretching vibration at $1658 \mathrm{~cm}^{-1}$, and amide II band, associated with the $\mathrm{N}-\mathrm{H}$ bending vibration and C-N stretching vibration at $1542 \mathrm{~cm}^{-1}$, are known to be conformation sensitive. The differential amide I/II absorbance ratio and the broadening ratio of the amide I band between the cortex and the cuticle were obtained from the enlarged spectra in Figure 1(B) for the amide I and II regions, which should be related to the heterogeneity of the amino-acid composition and to the different secondary and tertiary structures in the cortex and the cuticle [12] [21]. In addition to these intense major bands from the backbone structures of the proteins, amide A has a clearly visible band at $3294 \mathrm{~cm}^{-1}$ (spectra to the left) due to the N-H stretching vibration.

The amino-acid composition of the cuticle differs from that of the cortex [12] [21]. The high contents of serine and proline in the cuticle are indications of a non-helical structure of the protein chain [23]. A broadening of the amide I band in the cuticle is also an indication of a non-helical structure [21]. The $\alpha$-helix structure of hair keratin is seen in the hair photos taken before beaching and after bleaching with hydrogen peroxide for $120 \mathrm{~min}$ and in the plot of brightness as a function of the bleaching exposure time to hydrogen peroxide. During the bleaching process, the cuticle lifts and is abraded, causing diffused reflection. Moreover, melanin and the disulfide bonds of keratin can be attacked by the hydrogen peroxide, leading to oxidative cleavage of the disulfide bonds. When the oxidation of the keratin matrix occurs in the cortex, where the melanin granules are mainly distributed, the melanin undergoes irreversible changes, resulting either in a toning down or a complete elimination of the color of the original hair fiber.

When the pristine hairs were dipped into 6\% hydrogen peroxide, bleaching occurred. In Figure 2(A), pictures before and after bleaching for $120 \mathrm{~min}$ are shown; the lowest bright point (darkest part) and least black part are marked with the brightness in the picture. Note that the brightness scale was initially determined by $(R, G, B)$ color values, ranging from $(0,0,0)$ to $(255,255,255)$ for the darkest black to the brightest white, respectively. Then a one dimensional grey-level (brightness) was calculated by the average of those three RGB values, from 0 black) to 255 (white). While the average brightness of 


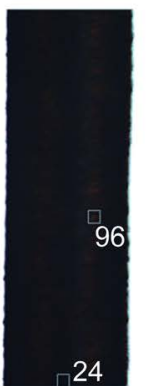

$0 \mathrm{~min}$

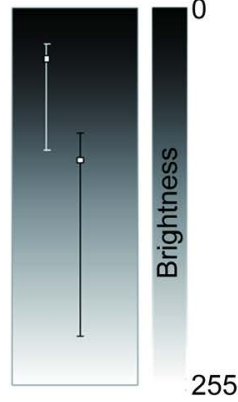

(A)

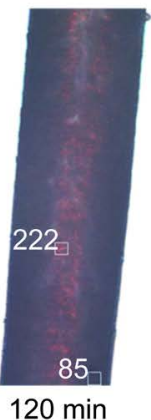

$120 \mathrm{~min}$

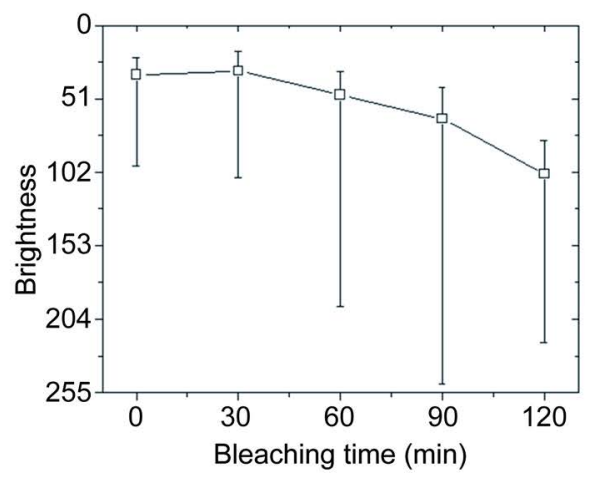

(B)

Figure 2. (A) Hair photos before $(0 \mathrm{~min})$ and after bleaching with hydrogen peroxide (120 min) and comparison of the brightness between the two hair photos on the black-white legend (0 - 255). Hair thickness was $\sim 88 \mu \mathrm{m}$. (B) Plot of the brightness as a function of the hydrogen-peroxide bleaching time.

non-bleached black hair was 34 , the minimum (darkest part) and the maximum (brightest part) values were 12 and 63, respectively; on the other hand, the average brightness of the hair bleached for 120 min was 103, and the minimum and the maximum values were 23 and 117, respectively. The numerical values for the brightness were obtained by using the black-to-white brightness scale given in the figure. The bleaching-time-dependent brightness of the hair, Figure 2(B), shows that the average brightness of the hair color increased from 34 to 103 with increasing bleaching time. The error bars in the plot indicate the maximum and the minimum values of the brightness. Thus, the average of brightness of the hair color was found to increase with increasing bleaching time.

Since the actual bleaching process, i.e., keratin oxidation and melanin elimination, is known to happen in the cortex, we focused on monitoring the chemical changes in the cortex during the bleaching process. The chemical images of amide $\mathrm{I}\left(1658 \mathrm{~cm}^{-1}\right)$ and amide $\mathrm{A}\left(3294 \mathrm{~cm}^{-1}\right)$ before $(0 \mathrm{~min})$ and after $(120 \mathrm{~min})$ bleaching the hair are shown in Figure 3(A), and their corresponding full spectra obtained at different etching times are shown in Figure 3(B). From the chemical image of amide I, the cortex region was clearly distinguished from the cuticle. In addition, the differences in the distributions of those bands before and after bleaching were negligible, which is in good agreement with the results in a previous report. Another noteworthy observation is that the cross-sectioned hair has a square-like shape after having been bleached for $120 \mathrm{~min}$, indicating that a severe macroscopic deformation had occurred in the hair during to the bleaching process.

A series of FT-IR spectra obtained for the region with the highest absorbance in the chemical image of amide I are shown in Figure 3(B). While the disulfide bond will appear around $500 \mathrm{~cm}^{-1}$, the bonds in the byproducts of disulfide oxidation of cysteine, i.e., cystinesulfonic acid, cystine monoxide, and cystine dioxide, should appear as characteristic infrared absorptions at 1040, 1072, and $1125 \mathrm{~cm}^{-1}$, respectively [12] [14] [22]. Figure 3(C) shows the absorbance contour maps of the cystine-monoxide band at 1072 


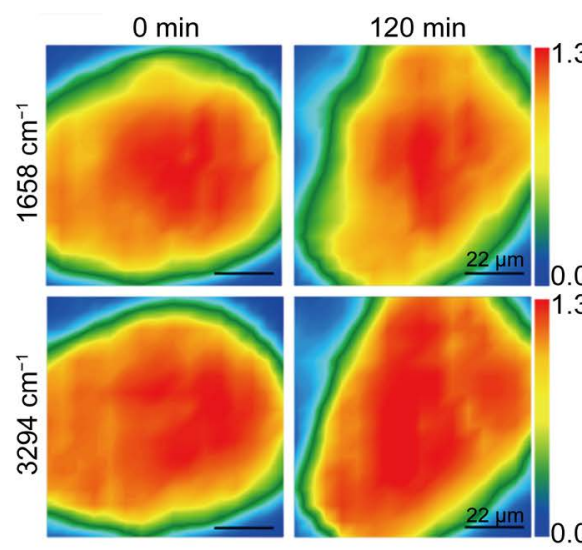

(A)

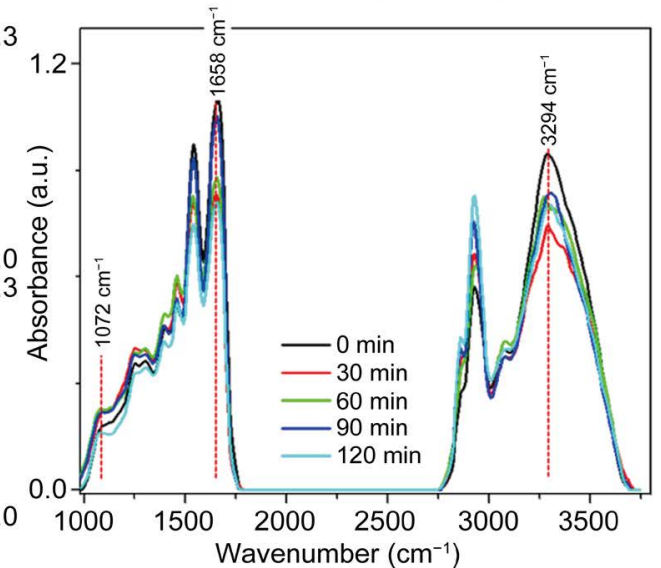

(B)
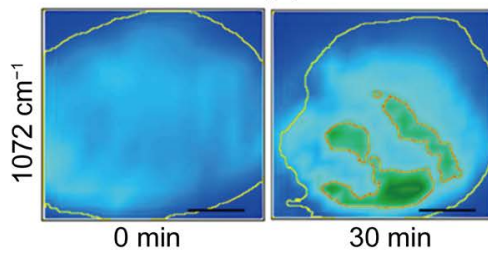

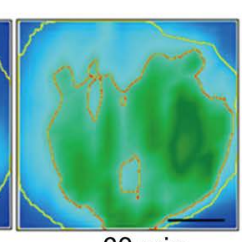

$60 \mathrm{~min}$

(C)

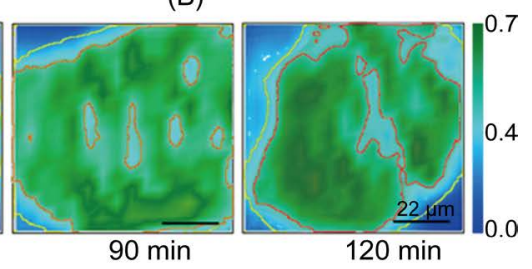

Figure 3. (A) Chemical images of amide I $\left(1658 \mathrm{~cm}^{-1}\right)$ and amide $\mathrm{A}\left(3294 \mathrm{~cm}^{-1}\right)$ for the hair before $(0 \mathrm{~min})$ and after $(120 \mathrm{~min})$ bleaching. (B) Bleaching-time-dependent micro-spectra from the highest absorbance in the chemical image of amide I. Bands at 1072, 1659, and $3294 \mathrm{~cm}^{-1}$ were present in the chemical images and were assigned to cystine monoxide, amide $\mathrm{I}$, and amide A, respectively. (C) Images of the cystine-monoxide band (green area) at $1072 \mathrm{~cm}^{-1}$ for different bleaching times.

$\mathrm{cm}^{-1}$ for different bleaching times, detailing how the oxidation process is spread throughout the cortex. In the first image (before bleaching), no hint of the cysteinemonoxide band is observed in the cortex. With increasing bleaching time, the band for cystine monoxide becomes more intense and progressively occupies larger areas in the cortex. Intense absorbance spots first appear at $1072 \mathrm{~cm}^{-1}$ for a region of the cortex interfacing with the cuticle in the hair bleached for $30 \mathrm{~min}$. The areas having strong absorbance for cysteine monoxide are spread throughout the cortex. Presumably, the hydrogen peroxide that had permeated from the cuticle penetrated the cortex region. After 90 min of bleaching, the absorbance band due tocystine monoxide can be seen to cover the entire cross-sectioned hair, indicating that the entire hair, from the cuticle to the cortex, was significantly attacked by hydrogen peroxide. Although this observation may not necessarily correlate with the actual color change upon bleaching, we can, nevertheless, conclude that 1 ) the cleavage and oxidation of disulfide bonds is complete within the first 90 minutes of hydrogen-peroxide treatment, and 2) the formation of cystine derivatives does not affect the protein's tertiary structure (i.e., keratin's helical structure) significantly.

In Figure 4, we plot the area and the intensity ratios of cystine monoxide $\left(1072 \mathrm{~cm}^{-1}\right)$ to amide $\mathrm{I}\left(1658 \mathrm{~cm}^{-1}\right)$ as functions of the bleaching time. The area ratio is observed to increase rapidly for early bleaching times $<60 \mathrm{~min}$ and to become saturated after 60 


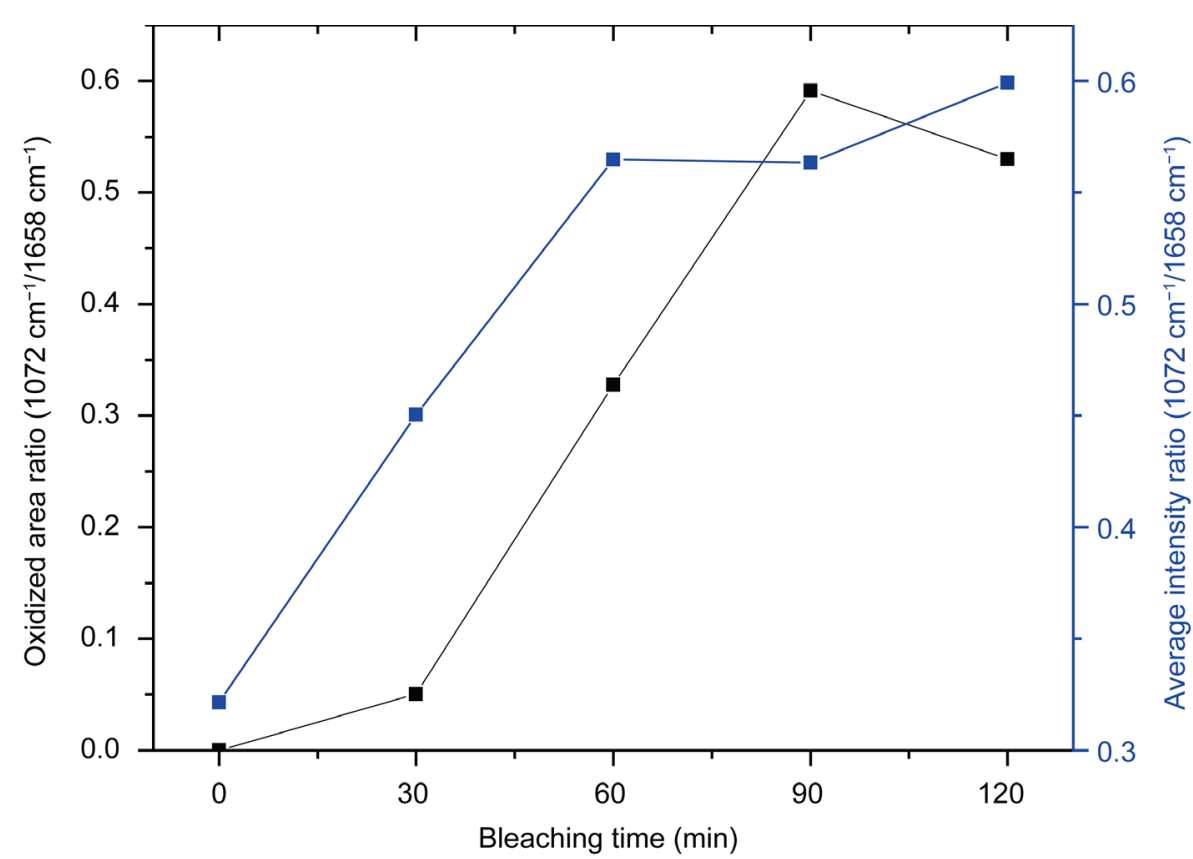

Figure 4. Plots of the average intensity and the oxidized area ratios of the cystine-monoxide band to the amide I band $\left(1072 \mathrm{~cm}^{-1} / 1658 \mathrm{~cm}^{-1}\right)$ as functions of the bleaching time.

minutes. From this, we infer that diffusion of the oxidation agent (here, hydrogen peroxide) throughout the cortex happens somewhat quickly, providing partial cleavage of some disulfide bonds. Meanwhile, the absorbance ratio showed a delayed increase after 30 minutes of bleaching, indicating that some bonds are hidden along the diffusion path and require a longer time to access.

\section{Conclusion}

The FT-IR microspectroscopic imaging method enables chemical imaging by combining spectral and spatial information. The chemical images contain chemical information from all pixels covering the measured areas at the same time. It is also a useful method for the analysis of human hair because it can be used to analyze both the cortex and the cuticle of cross-sectioned hair. In this research, we studied the bleaching of hair and measured the chemical effects of bleaching as a function of position in the crosssectioned hair. Transformations due to the breakage of bonds in specific chemical groups were observed and spatially characterized. The cystine-monoxide band, which was due to the products of disulfide oxidation of the amino acid cystine, was associated with hair damage. By spectrally mapping infrared functional groups, we could demonstrate how the oxidative reaction spread throughout the cortex as a function of the bleaching time.

\section{Acknowledgements}

This work was supported by the Mid-Career Researcher Program (2016R1A2B3015239), and the Leading Foreign Research Institute Recruitment Program (2013K1A4A3055268) 
through the National Research Foundation of South Korea funded by the Ministry of Science, ICT \& Future Planning, South Korea.

\section{References}

[1] Cruz, C.F., Fernandes, M.M., Gomes, A.C. Coderch, L., Marti, M., Mendez, S., Gales, L., Azoia, N.G., Shimanovich, U. and Cavaco-Paulo, A. (2004) Keratins and Lipids in Ethnic hair. International Journal of Cosmetic Science, 35, 244-249.

http://dx.doi.org/10.1111/ics.12035

[2] Robbins, C.R. (2002) Chemical and Physical Behavior of Human Hair. 4th Edition, Springer-Verlag, New York.

[3] Wolfram, L.J., Hall, K. and Hui, I. (1970) The mechanism of hair bleaching.Journal of the Society of Cosmetic Chemistry, 21, 875-900.

[4] Muro, C.K., Doty, K.C., Bueno, J., Halamkova, L. and Lednev, I.K. (2015) Vibrational Spectroscopy: Recent Developments to Revolutionize Forensic Science. Analytical Chemistry, 87, 306-327. http://dx.doi.org/10.1021/ac504068a

[5] Marcott, C., Lo, M., Kjoller, K., Fiat, F., Baghdadli, N., Balooch, G. and Luengo, G.S. (2014) Localization of Human Hair Structural Lipids Using Nanoscale Infrared Spectroscopy and Imaging. Applied Spectroscopy, 68, 564-569. http://dx.doi.org/10.1366/13-07328

[6] Espinoza, E.O., Baker, B.W., Moores, T.D. and Voin, D. (2008) Forensic Identification of Elephant and Giraffe Hair Artifacts Using HATR FTIR Spectroscopy and Discriminant Analysis. Endangerd Species Research, 9, 239-246. http://dx.doi.org/10.3354/esr00125

[7] Bueno, J. and Lednev, I.K. (2014) Attenuated Total Reflectance-FT-IR Imaging for Rapid and Automated Detection of Gunshot Residue. Analytical Chemistry, 86, 3389-3396. http://dx.doi.org/10.1021/ac4036718

[8] Kakoulli, I., Prikhodko, S.V., Fischer, C., Cilluffo, M., Uribe, M., Bechtel, H.A., Fakra, S.C. and Marcus, M.A. (2014) Distribution and Chemical Speciation of Arsenic in Ancient $\mathrm{Hu}-$ man Hair Using Synchrotron Radiation. Analytical Chemistry, 86, 521-526. http://dx.doi.org/10.1021/ac4024439

[9] Lyman, D.J. and Murray-Wijelath, J. (2005) Fourier Transform Infrared Attenuated Total Reflection Analysis of Human Hair: Comparison of Hair from Breast Cancer Patients with Hair from Healthy Subjects. Applied Spectroscopy, 59, 26-32.

http://dx.doi.org/10.1366/0003702052940440

[10] Lyman, D.J. and Fay, S.G. (2014) The Effect of Breast Cancer on the Fourier Transform Infrared Attenuated Total Reflection Spectra of Human Hair. Ecancer, 8, 405-413. http://dx.doi.org/10.3332/ecancer.2014.405

[11] Wang, X., Qi, Z., Liu, X., Wang, S., Li, C., Liu, G., Xiong, Y., Li, T., Tao, J. and Tian, Y. (2010) The Comparison of Hair from Gastric Cancer Patients and from Healthy Persons Studied by Infrared Microspectroscopy and Imaging Using Synchrotron Radiation. Cancer Epidemiology, 34, 453-456. http://dx.doi.org/10.1016/j.canep.2010.03.016

[12] Bantignies, J.-L., Carr, G.L., Lutz, D., Maruli, S., Williams, G.P. and Fuchs, G. (2000) Chemical Imaging of Hair Infrared Microspectroscopy Using Synchrotron Radiation. Journal of Cosmetic Science, 51, 73-90.

[13] Ha, B.-J. (2008) Instrumental Analysis of the Human Hair Damaged by Bleaching Treatments. Journal of Fashion Business, 12, 23-33.

[14] Kon, R., Nakamura, A. and Takeuchi, K. (1998) Artificially Damaged Hairs: Preparation and Application for the Study of Preventive Ingredients. International Journal of Cosmetic 
Science, 20, 369-380. http://dx.doi.org/10.1046/j.1467-2494.1998.177060.x

[15] Signori, V. and Lewis, D.M. (1997) FTIR Investigation of the Damage Produced on Human Hair by Weathering and Bleaching Processes: Implementation of Different Sampling Techniques and Data Processing. International Journal of Cosmetic Science, 19, 1-13. http://dx.doi.org/10.1111/j.1467-2494.1997.tb00161.x

[16] Mendelsohn, R., Rerek, M.E. and Moore, D.J. (2000) Infrared Spectroscopy and Microscopic Imaging of Stratum Corneum Models and Skin Invited Lecture. Physical Chemistry Chemical Physics, 2, 4651-4657. http://dx.doi.org/10.1039/b003861j

[17] Lau, K., Hedegaard, M.A.B., Kloepper, J.E., Paus, R., Wood, B.R. and Deckert, V. (2011) Visualization and Characterization of Defined Hair Follicle Compartments by Fourier Transform Infrared (FTIR) Imaging without Labelling. Journal of Dermatological Science, 63, 191-198. http://dx.doi.org/10.1016/j.jdermsci.2011.05.002

[18] Kazarian, S.G. and Chan, K.L.A. (2013) ATR-FTIR Spectroscopic Imaging: Recent Advances and Applications To Biological Systems. Analyst, 138, 1940-1951.

http://dx.doi.org/10.1039/c3an36865c

[19] Kong, R. and Bhargava, R. (2011) Characterization of Porcine Skin as a Model for Human Skin Studies Using Infrared Spectroscopic Imaging. Analyst, 136, 2359-2366. http://dx.doi.org/10.1039/c1an15111h

[20] Thibaut, S., de Becker, E., Bernard, B.A., Huart, M., Fiat, F., Baghdadli, N., Luengo, G.S., Leroy, F., Angevin, P., Kermoal, A.M., Muller, S., Peron, M., Provot, G., Kravtchenko, S., Saint-Leger, D., Desbois, G., Gauchet, L., Nowbuth, K., Galliano, A., Kempf, J.Y. and Silberzan, I. (2010) Chronological Ageing of Human Hair Keratin Fibres. International Journal of Cosmetic Science, 32, 422-434. http://dx.doi.org/10.1111/j.1468-2494.2009.00570.x

[21] Jurdana, L.E., Ghiggino, K.P., Leaver, I.H. and Cole-Clarke, P. (1995) Application of FT-IR Step-Scan Photoacoustic Phase Modulation Methods to Keratin Fibers. Applied Spectroscopy, 49, 361-366. http://dx.doi.org/10.1366/0003702953963508

[22] Panayiotou, H. and Kokot, S. (1999) Matching and Discrimination of Single Human-Scalp Hairs by FT-IR Micro-Spectroscopy and Chemometrics. Analytica Chimica Acta, 392, 223235. http://dx.doi.org/10.1016/S0003-2670(99)00230-5

[23] Wolfram, L.J. and Lindermann, M.K.O. (1971) Some Observations on the Hair Cuticle. Journal of the Society of Cosmetic Chemistry, 22, 839-850.

\section{Submit or recommend next manuscript to SCIRP and we will provide best service for you:}

Accepting pre-submission inquiries through Email, Facebook, LinkedIn, Twitter, etc.

A wide selection of journals (inclusive of 9 subjects, more than 200 journals)

Providing 24-hour high-quality service

User-friendly online submission system

Fair and swift peer-review system

Efficient typesetting and proofreading procedure

Display of the result of downloads and visits, as well as the number of cited articles

Maximum dissemination of your research work

Submit your manuscript at: http://papersubmission.scirp.org/

Or contact jcdsa@scirp.org 